\title{
Review:
}

\section{Treatment of diabetes with encapsulated pig islets: an update on current developments*}

\author{
Hai-tao ZHU ${ }^{\dagger 1,2}$, Lu LU ${ }^{2}$, Xing-yu LIU ${ }^{3}$, Liang YU ${ }^{2}$, Yi LYU ${ }^{2,4}$, Bo WANG ${ }^{\dagger 2}$ \\ $\left({ }^{1}\right.$ Heart Center, Northwest Women's and Children's Hospital, Xi'an 710061, China) \\ ( ${ }^{2}$ Department of Hepatobiliary Surgery, the First Affiliated Hospital, Medical College, Xi'an Jiaotong University, Xi'an 710061, China) \\ ( ${ }^{3}$ Department of Hand Surgery, China-Japan Union Hospital, Norman Bethune Health Science Center, Jilin University, Changchun 130033, China) \\ ( ${ }^{4}$ Institute of Advanced Surgical Technology and Engineering, Xi'an Jiaotong University, Xi' an 710061, China) \\ †E-mail: zht0915@163.com; bobwang75@yeah.net
}

Received Nov. 16, 2014; Revision accepted Feb. 8, 2015; Crosschecked Apr. 9, 2015

\begin{abstract}
The potential use of allogeneic islet transplantation in curing type 1 diabetes mellitus has been adequately demonstrated, but its large-scale application is limited by the short supply of donor islets and the need for sustained and heavy immunosuppressive therapy. Encapsulation of pig islets was therefore suggested with a view to providing a possible alternative source of islet grafts and avoiding chronic immunosuppression and associated adverse or toxic effects. Nevertheless, several vital elements should be taken into account before this therapy becomes a clinical reality, including cell sources, encapsulation approaches, and implantation sites. This paper provides a comprehensive review of xenotransplantation of encapsulated pig islets for the treatment of type 1 diabetes mellitus, including current research findings and suggestions for future studies.
\end{abstract}

Key words: Encapsulation, Pig, Islet, Xenotransplantation, Diabetes mellitus

doi:10.1631/jzus.B1400310 Document code: A

CLC number: R657.5

\section{Introduction}

Pancreatic islet transplantation is a viable and attractive option for the treatment of type 1 diabetes mellitus (T1DM) (Shapiro et al., 2000; Leitão et al., 2008; Hatziavramidis et al., 2013; Ramesh et al., 2013). Due to a shortage of human donors, the sourcing of xenogenic islets from pig donors has emerged as an alternative strategy for transplantation. The pig is considered as the suitable islet donor candidate with advantages of the structural similarity between the insulin of pigs and humans, the lack of amyloid formation, resistance to recurrent autoim-

\footnotetext{
${ }^{\ddagger}$ Corresponding author

* Project supported by the National Natural Science Foundation of China (No. 30700772)

(ib) ORCID: Hai-tao ZHU, https://orcid.org/0000-0003-0867-1354

(c) Zhejiang University and Springer-Verlag Berlin Heidelberg 2015
}

munity, and feasibility for genetic immunomodulation (Koulmanda et al., 2003; Yonekawa et al., 2005; Potter et al., 2010; Wynyard et al., 2014; Zhu et al., 2014a; 2014b). Recently, the demonstration of sustained diabetes reversal and prolonged survival of islet grafts (from wild-type or genetically modified pigs) in immunosuppressed diabetic non-human primates (NHPs) (van der Windt et al., 2009; Thompson et al., 2011a; 2011b; 2012; Bottino et al., 2014) has signified a major step in the advance of the use of pig islets as a promising cellular therapy for the treatment of T1DM. However, due to immune incompatibility, xenogeneic rejection is still a major challenge in the application of pig islet xenotransplantation (Scalea et al., 2012). At present, the mainstay of immune-modulatory remedies is the use of heavy and permanent immunosuppressants which have been shown to have harmful effects on both 
recipients (e.g. opportunistic infection, malnutrition, neuritis, and severe morbidity) and grafts (e.g. islet toxicity). To promote pig islet xenotransplantation into clinical trials safely and effectively, the problems of life-long immunosuppressive agents must first be overcome (Cooper and Casu, 2009; O'Connell et al., 2013; Shin et al., 2014). Indeed, only preclinical studies in which the NHP recipients do not need continuous immunosuppressive therapy are considered as an acceptable and possible basis for a clinical trial (Cooper and Casu, 2009). Immunoisolation, hiding the grafts from the recipients' immune system, fundamentally differs from the conventional strategy of continuing immunosuppressive/tolerance-inducing treatments, and represents a potential and appropriate approach for effectively reducing the immunological barriers to the use of pig islets and for promoting graft survival and functionality (Dufrane et al., 2006b; Elliott et al., 2007; Zimmermann et al., 2007; Dufrane and Gianello, 2012; Sakata et al., 2012).

Immunoisolation is usually achieved by coating islet grafts with semi-permeable membranes consisting of polymer materials, creating what are often referred to as an artificial pancreas (AP) or an encapsulated islet (O'Sullivan et al., 2011). The semipermeable, bio-compatible membranes facilitate the exchange of oxygen, nutrients, insulin, and waste, but protect the islet grafts from the host immune response. With the development of more stable and biocompatible encapsulation systems, pig islet xenografts are able to survive and release insulin for a prolonged period of time, thereby controlling glucose metabolism with a reduction or even the omission of immunosuppressive medication (Table 1). Xenotransplantation of encapsulated pig islets offers the prospect of a practical treatment for insulin-dependent diabetes mellitus (IDDM) (Dufrane and Gianello, 2012; Ramesh et al., 2013; Zhu et al., 2014b). However, several important issues need to be addressed before large scale application can be proposed, for example, seeking or generating a reliable and transplantable source of pig islets/insulinproducing cells, developing an encapsulation approach/ technology suitable for large-clinical application, and determining suitable implant sites to facilitate islet engraftment and function. The purpose of this article is to provide a comprehensive review of these topics of pig islet encapsulation.

\section{Sources of pig islets}

High-quality pig islets contribute a lot to the efficacy and functional duration of an AP. Compared with other variables such as gender and body-weight, donor age seems to have a greater impact on islet size, yield, and functionality (Dufrane et al., 2005; Bottino et al., 2007; Kim et al., 2009). Neonatal pig pancreatic cell clusters (NPCCs) provide additional advantages over adult pig islets (APIs), such as their ease of isolation and purification, resistance to ischemia and inflammation during preparation, low cost, and low level of T-cell response (Nagaraju et al., 2015; Zhu et al., 2014a). After implantation, encapsulated immature pig islets, including NPCCs and fetal pig islet-like cell clusters (FPICCs), can proliferate and differentiate into mature $\beta$-cell masses and show excellent metabolic control in vivo (Omer et al., 2003b; Foster et al., 2007). Unlike APIs, immature pig islet cells are resistant to the toxic effects of proinflammatory cytokines, including tumor necrosis factor- $\alpha$, interleukin- $1 \beta$, and interferon- $\gamma$ (Bai et al., 2002), which may diffuse freely across the hydrogel membranes of the capsules due to their low molecular weight. NPCCs offer an alternative and promising source for islet encapsulation in both preclinical and clinical xenotransplantation (Table 1).

In consideration of the potential risk of zoonosis, islet xenografts should be obtained from specific pathogen-free (SPF) and designated pathogen-free (DPF) pig breeds. Chicago Medical School miniature pigs, New Zealand Auckland Island pigs, and transgenic pigs targeting porcine endogenous retrovirus (PERV) (Elliott et al., 2000; Kim et al., 2007; Semaan et al., 2012; Wynyard et al., 2014) represent available donor sources for islet immunoisolation. The genetic modification of islet cells is also a practical method for generating new transplantable grafts with special resistances. For example, the immunoisolation of APIs genetically modified to overexpress antiapoptotic $B c l-2$ gene could significantly reduce islet loss after intraportal infusion (Contreras et al., 2004). The successful application of combined encapsulation and genetic modification technologies opens up a new strategy to improve the outcomes from xenoislet transplantation. In the future, genetically engineered pigs (e.g. transgenic pigs expressing human complement regulators, human heme oxygenase-1, or 


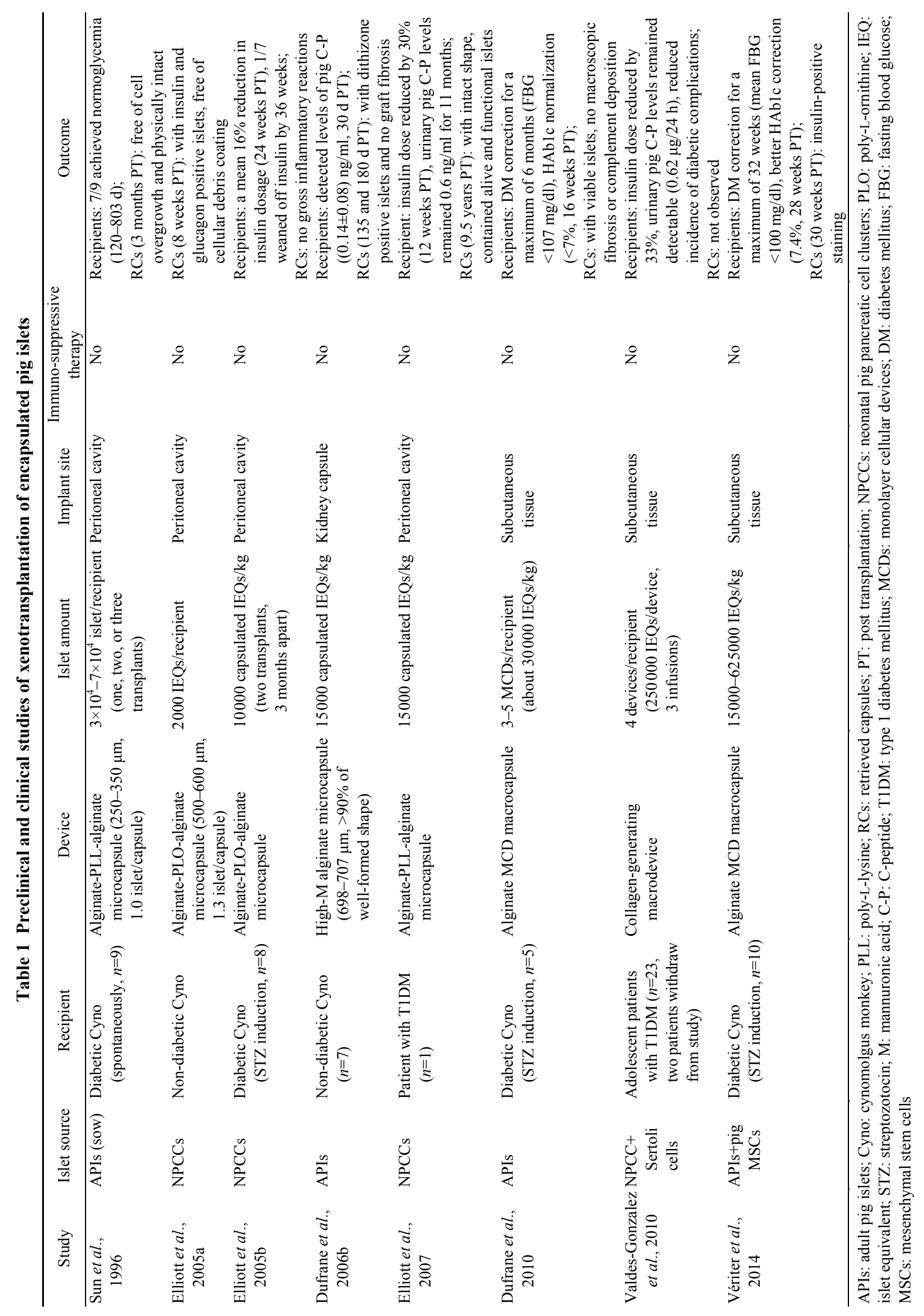




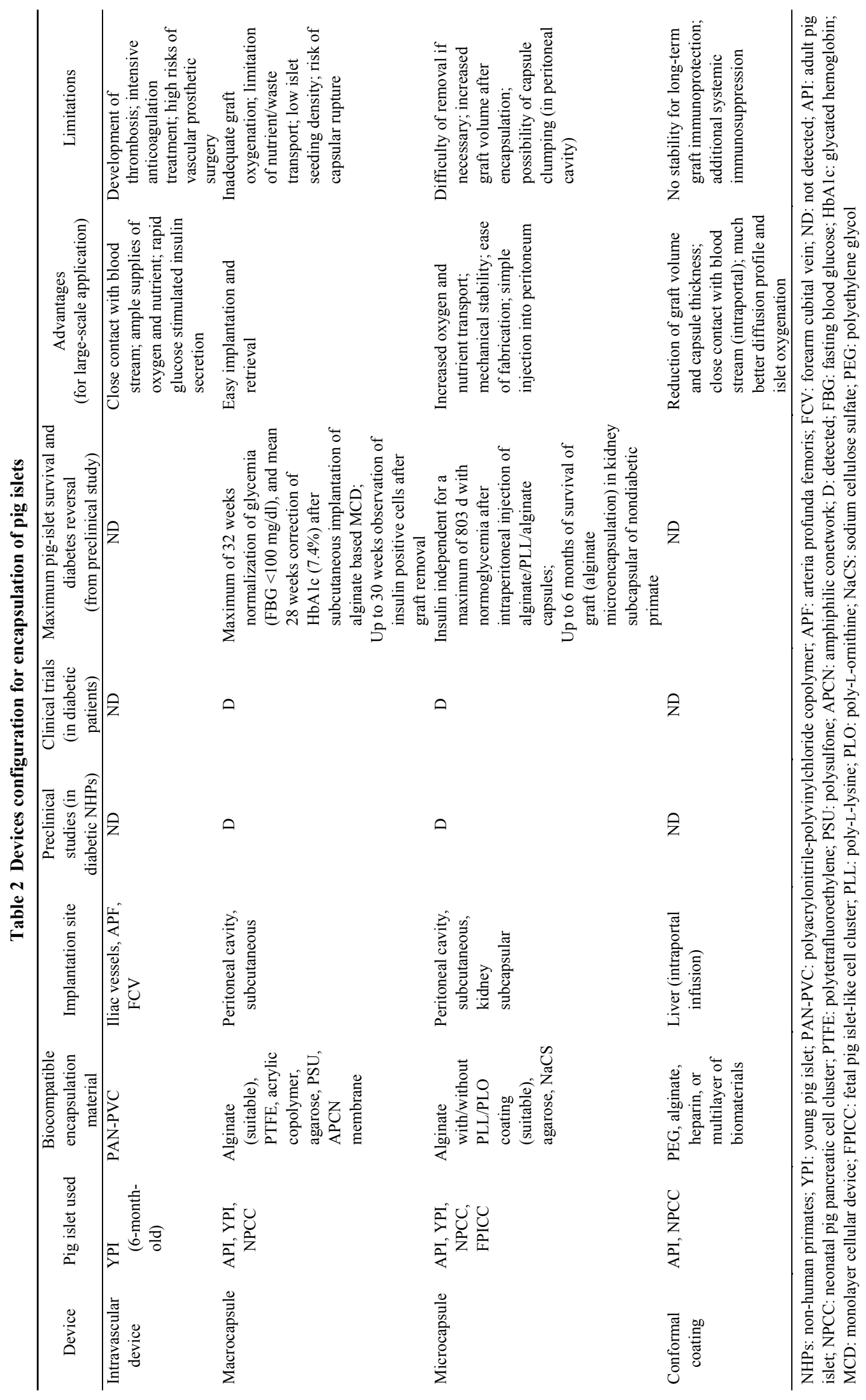


knocking-out tissue factors, or multi-transgenic pigs) will emerge as promising donor sources for islet immunoisolation in preclinical and clinical applications with advantages of low antigenicity, resistance to inflammation or complement mediated islet damage or loss, and sustained islet survival and functionality.

\section{Encapsulation approaches}

The introduction of the concept of immunoisolation dates back to 1933. Since then, several different types of immunoisolation devices have been created and studied (Table 2). Overall, the designs of encapsulation systems can be divided into two major categories: intravascular and extravascular devices.

\subsection{Intravascular devices}

In intravascular devices, islet grafts are enclosed in a large semi-permeable chamber containing a number of small diameter artificial capillaries made of polyacrylonitrile-polyvinylchloride copolymer (PAN-PVC) (Lanza et al., 1996; Borg and Bonifacio, 2011). For implantation, this device is connected directly to the circulatory system of the recipient by vascular anastomosis. The close contact between the islets and the host's blood stream ensures a rapid exchange of insulin and glucose, thereby inducing a strict and prompt regulation of blood glucose (BG). In diabetic dogs, intravascular devices containing pig islets (160000-430000 islet equivalents (IEQs)/device) provided good glycemic control for more than eight months without immunosuppression (Maki et al., 1996). However, thrombus formation in the lumen of the intravascular device or at the anastomotic site proved to be a major obstacle, in spite of anticoagulant therapy in massive doses. Also, it is quite plausible that the relatively high flow-rates through the device could not allow an adequate exchange of nutrients to sustain the prolonged survival and favorable function of islet grafts. Complications (e.g. bleeding, intimal hyperplasia, and infection) associated with vascular prosthetic surgery still remain a serious threat limiting the therapeutic potential of this approach. Thus, research on intravascular devices has remained on hold since the 1990s (Petruzzo et al., 1991; Maki et al., 1996; Maki and Monaco, 1997). But in 2008,
Prochorov et al. (2008) conducted an intravascular implantation of fetal rabbit islets (>6000 IEQs $/ \mathrm{kg}$ ) contained in nylon microporous macrocapsules into the arterial-venous fistulas of T1DM patients without immunosuppression. All recipients were given the current standard antithrombotic therapy. After two years of follow-up, the authors reported a significant reduction in exogenous insulin demands, together with increases in C-peptide and immunoreactive insulin levels in 14 of the 19 patients. Moreover, neither vascular lumen narrowing nor thrombosis was observed. This encouraging achievement raises the prospect that new biomaterials with new intravascular approaches may lead to good outcomes, which will produce a clinically relevant intravascular device.

\subsection{Extravascular devices}

This type of device does not require anastomosis when it is transplanted into the recipient and therefore has an advantage over intravascular devices in terms of preclinical and clinical applications (Table 2). Usually, extravascular devices are categorized into two main types by their sizes: macroencapsules (as large as $3 \mathrm{~cm} \times 8 \mathrm{~cm}$ ) and microencapsules (ranging from 150 to $1000 \mu \mathrm{m}$ ) (de Vos et al., 2010; Buder et al., 2013).

\subsubsection{Macrocapsules}

Macrocapsules contain large numbers of islet grafts within a tubular diffusion chamber or planar chamber. One advantage of macrocapsules is that they can be implanted and removed with minimal risk. On the other hand, their major drawback is the limited oxygen diffusion and nutrient transport, which tends to result in impaired viability, dysfunction, or even central necrosis in islets (Beck et al., 2007; Weir, 2013). Current research on macroencapsulation systems focuses largely on the development of techniques and configurations, which can promote neovascularization and provide sufficient oxygen and nutrition for islet cells (Grundfest-Broniatowski et al., 2009; Dufrane et al., 2010; Barkai et al., 2013; Vériter et al., 2014; Scharp and Marchetti, 2014).

\subsubsection{Current approaches}

A commonly used commercial macrocapsule is the TheraCyte device, which is made of bilayered polytetrafluoroethylene (PTFE) membranes (Sörenby 
et al., 2008; Malavasi et al., 2010; Kirk et al., 2014). The outer membrane of the TheraCyte device is designed for strength and to facilitate neovascularization, and the inner membrane provides immune protection. In diabetic mice, subcutaneous transplantation of NPCCs encapsulated in the TheraCyte device greatly reversed diabetes for up to 10 weeks. In non-diabetic monkeys, histology of the retrieved device showed that it had no coating of cellular debris and no inflammatory reaction was observed in the adjacent tissues (Elliott et al., 2005a). The TheraCyte device is impermeable to immune cells, but the pore size suggests that the membrane may be permeable to antibodies and complements. The absence of pig islet damage inside the device can possibly be explained by the slow passage of IgG antibodies and little expression of $\alpha$ galactose antigens (activators of acute rejection) on islet cells (Kin et al., 2000; Marigliano et al., 2011; Kumagai-Braesch et al., 2013). Furthermore, a novel implantable macrochamber ( $\beta$-Air device) has been created to offer immune protection and an adequate oxygen supply for islet grafts (Ludwig et al., 2010; Barkai et al., 2013). This disc-shaped combinational device consists of two compartments, an oxygen supply compartment and an immune protected compartment containing islet grafts immobilized in alginate hydrogel. Ludwig et al. (2010) demonstrated that API allografts remained morphologically intact, viable, and functional for significant times within the double-chambered bioreactor connected to subcutaneous refueling ports through which an oxygen- $\mathrm{CO}_{2}$ mixture was delivered by daily injection. With structural improvements (e.g. increased islet mass (up to $(4160 \pm 380)$ IEQs $/ \mathrm{cm}^{2}$ ), a better gas ventilation system, and an improved immune barrier) and successful applications in large animals (Ludwig et al., 2013; Neufeld et al., 2013), the $\beta$-Air device provides a potential alternative strategy for preclinical pig islet xenotransplantation. Nevertheless, the primary obstacle is that the cell density in this device needs to be quite low to ensure an adequate oxygen supply. This indicates that if large numbers of pig islets $(25000-100000 \mathrm{IEQs} / \mathrm{kg})$ are required to achieve insulin independence in diabetic NHPs (Hering et al., 2006; Casu et al., 2008; van der Windt et al., 2009; Thompson et al., 2011a; 2012; Zhu et al., 2014a), numerous or larger devices must be implanted. However, it is impossible to find a suitable surgical site to accommodate such macrodevices. The challenge will be settled if approaches can be found to offer more oxygen by improved delivery methods or better vascularisation.

More recently, a monolayer configuration (made of alginate) of macroencapsulated APIs implanted subcutaneously showed the ability to correct hyperglycemia for up to six months in diabetic monkeys without immunosuppression (Dufrane et al., 2010). In this device, pig islets were seeded as a monolayer on a human decellularized collagen matrix (mean 50000 IEQs $/ \mathrm{cm}^{2}$ ) to improve the number of islets per unit surface area and to enhance biological support (e.g. oxygen delivery and nutrient exchange). Subsequently, a better and longer diabetic control (up to 32 weeks) was obtained in diabetic monkeys after subcutaneous implantation of APIs and mesenchymal stem cells (MSCs) which were co-encapsulated in the same monolayer device (Vériter et al., 2014). The co-transplantation of MSCs significantly improved the vascularization (neoangiogenesis) and oxygenation of the macrodevice in terms of an increased number of vessels and elevated generation of vascular endothelial growth factor (VEGF). Also, the pig MSCs could be expanded and differentiated, therefore potentially constituting an alternative, renewable, and continuous source of insulin-producing cells. A phase I clinical trial is ongoing in Belgium to further investigate the safety and effectiveness of this monolayer cellular device for allotransplantation of encapsulated islets into humans.

\subsubsection{Clinical trials}

Valdés-González et al. (2005) reported acceptable clinical outcomes from xenotransplantation of NPCCs encapsulated in an autologous collagengenerating device with homologous Sertoli cells, which had excellent immunomodulatory properties. Prior to islet-Sertoli cell infusion (30-100 Sertoli cells per islet), the macrodevices $(6 \mathrm{~cm} \times 0.8 \mathrm{~cm})$ consisting of two steel mesh tubes and a PTFE rod interior were implanted subcutaneously for two months to allow tissue ingrowth and vascularization. Without the use of any immunosuppressants, 6 of 12 patients showed a $50 \%$ or greater reduction in exogenous insulin requirements, and this reduction was maintained for up to four years. Three years postimplantation, histological samples of tissue extracted 
from the device stained positive for insulin-producing cells. Moreover, routine microbiological screening of the recipients was constantly negative. With technical improvements, Valdés-González et al. (2007) further demonstrated the safety and feasibility of this xenotransplantation procedure for the control of T1DM in a single detailed case study. Valdes-Gonzalez et al. (2010) reported a longitudinal study of 23 T1DM patients xenotransplanted with collagen macroencapsulated NPCC-Sertoli cells between 2000 and 2004. All recipients produced detectable porcine C-peptide in their urine and more than $50 \%$ of recipients presented a greater reduction $(>33 \%)$ in their exogenous insulin requirement. Unfortunately, this pilot study did not contain a diabetic control undergoing tightly controlled treatment to validate the results.

\subsubsection{Some considerations}

Although interesting data have come from macroencapsulation studies, additional issues have to be addressed before the macroencapsulation of pig islets can emerge as a practical clinical option for the treatment of T1DM. (1) Macrocapsules with optimal geometry and structure are needed to enhance the supply of oxygen and nutrients for the contained pig islets, and to minimize the diffusion distance for insulin and glucose. (2) Studies have demonstrated that macroencapsulation of pig islets can result in normoglycemia in diabetic recipients; however, very little is known about the kinetics of insulin release from the encapsulated pig islets. Is there an adequate and timely insulin response to changes in glucose levels? (3) Vascularization of the membrane, a mandatory process for favorable function and prolonged survival of macroencapsulated islets, is preceded by inflammation which involves the recruitment of inflammatory cells around the macrodevices and the formation of an extracellular matrix to facilitate ingrowth of endotheliocytes (de Vos et al., 2010). It is essential to explore which factors need protection in a macrodevice during the early and late implant stages. (4) What is the optimal amount of pig islet grafts that can be well supported by a certain surface (i.e. the optimal packing density of islet cells) that receives oxygen and nutrient supply from the peripheral tissues?

\subsubsection{Microcapsules}

Microcapsules incorporate individual or small groups of islets in a spherical hydrogel polymer with a stable mechanical structure. A number of considerations favor microcapsules over macrocapsules (Table 2). The spherical geometry and low volume of microcapsules offer better oxygen and nutrient transport due to a higher surface area-to-volume ratio (van Schilfgaarde and de Vos, 1999; Beck et al., 2007). Furthermore, microcapsules require less complex or expensive manufacturing procedures, and can be simply injected without major surgery (Scharp and Marchetti, 2014). Their primary drawback is that it is difficult to remove them completely, especially if there is pericapsular fibrotic overgrowth (PFO) after implantation. Nevertheless, a novel method, attachment of microcapsules to a plasma-treated polydimethylsiloxane (PDMS) sheet, appears to be applicable for retrieving microencapsulated pig islets when required (Shin et al., 2013).

\subsubsection{Current approaches}

Currently, there seems to be a great deal more researches and developments on microdevices than on macrodevices in preclinical pig islets xenotransplantation (Table 1). In this area, alginate is the most suitable and commonly used biomaterial to entrap islet cells (de Vos et al., 2014; Orlando et al., 2014) (Tables 1 and 2). In general, islet-containing alginate solution is dropped through a nozzle into calcium or barium solution to generate a microbead incorporating an individual or few islet grafts. The surface of the device can be further coated with polycation/ alginate to confer better permselectivity and mechanical stability. Moreover, a variety of capsule sizes and structures are available, including a coreshell version, double core/shell version, and a solidlike/liquid core version. However, a lack of standardized formulations contributes greatly to current reported lab-to-lab variation in biocompatibility and immune-protection of microencapsulation systems.

Several studies have demonstrated the successful protection of alginate-microencapsulated pig islets against immune destruction and long-term reversal or control of hyperglycemia (ranging from four months to over $450 \mathrm{~d}$ ) in diabetic rodents (Lanza et al., 1999; Omer et al., 2003b; Foster et al., 2007; Cui et al., 
2009). More importantly, studies describing xenotransplantation of pig islets (APIs or NPCCs) microencapsulated in an alginate-matrix confirmed their biocompatibility and safety (no evidence of porcine viral transmission) in nondiabetic NHPs (Elliott et al., 2005a; Dufrane et al., 2006b), and demonstrated their capacity to achieve metabolic control and reduce insulin requirements in diabetic dogs and NHPs (Sun et al., 1996; Elliott et al., 2005b; Abalovich et al., 2009). However, the clinical application of microencapsulated pig islets is not yet supported by more solid preclinical achievements. Only one historical study (Sun et al., 1996) indicated that spontaneously diabetic monkeys could become insulin independent for periods ranging from 120 to $803 \mathrm{~d}$ with normalized fasting BG levels following 1-3 transplants of microencapsulated APIs. Although the data obtained were encouraging for clinical practice, they might be largely dependent on the primate model. To achieve long-term biocompatibility and viability (e.g. $>6$ months) and favorable immunoprotection of alginate microencapsulated pig islets in primate recipients, several suggestions should be considered (Sun et al., 1996; Dufrane et al., 2006b; Calafiore and Basta, 2014): (1) the use of donor pigs with a well-defined genetic background; (2) the use of pig islets with high-purity ( $>90 \%$ purity); (3) fabrication of microcapsules using highly purified material with improved stability, low heavy metal, protein and endotoxin content, and a "clinical-grade" basic alginate is recommended; (4) the culture of microencapsulated islets in a medium containing $1.8 \mathrm{mmol} / \mathrm{L}$ $\mathrm{CaCl}_{2}$ for 18 or $24 \mathrm{~h}$ prior to implantation; and (5) the transplantation of grafts composed of more than $90 \%$ well-shaped capsules (of regular and spherical shape).

\subsubsection{Clinical trials}

Elliott et al. (2007) first reported a case of long-term survival ( $>9.5$ years) of microencapsulated NPCCs in a male patient with T1DM who received a single implantation of alginate based grafts (15000 IEQs $/ \mathrm{kg}$ ) into the peritoneal cavity in 1996. Following transplantation, the daily insulin dose was reduced by up to $30 \%$ and the urinary porcine C-peptide remained detectable for over one year. Ten years later, laparoscopic examination revealed living and functional NPCCs in his abdomen, and no evi- dence of xenosis or gross peritoneal reaction or fibrosis was observed. In 2007, a larger clinical study of commercial microencapsulated pig islets (also called "Diabecell") was conducted by the Living Cell Technologies (LCT) Company (Tan, 2010) (DIABECELL ${ }^{\circledR}$ is currently in late-stage clinical trials. Further information is available from http://www. lctglobal.com/products/diabecell/development-to-date). After implantation (5000-10000 IEQs $/ \mathrm{kg}$ ), two patients were completely independent of insulin administration for up to 32 weeks, and six patients displayed improved BG control as reflected by their reduced glycated haemoglobin (HbAlc) levels and daily insulin dose. Currently, other phase IIa trials are being conducted in New Zealand and Argentina.

\subsubsection{Some considerations}

All these clinical trials cannot be considered as true breakthroughs for the treatment of T1DM, since no exogenous insulin administration was interrupted and the metabolic control was not excellent. Nevertheless, these pilot clinical trials confirmed the safety and potential therapeutic effects of microencapsulated islet xenografts in human recipients. Improvements in microcapsule design and fabrication, coupled with the emergence of sufficient or renewable islet cell mass with low xenoantigenicity and high-quality (e.g. high purity, with inflammation- and hypoxia-resistance), as well as advances in optimized biomaterials and the bioengineering of implantation sites may help to provide a favorable and stable metabolic control in diabetic patients.

\subsection{Future directions: conformal coating}

Traditional methods of islet microencapsulation can still result in diffusional limitations associated with capsules of larger size ( $>600 \mu \mathrm{m}$ in diameter), which may lead to blunted insulin secretion in response to glucose, and even core hypoxia or necrosis of islets. In addition, the mean diameter of islets is about $150 \mu \mathrm{m}$, increasing the total volume of the implant by dozens of times after microencapsulation. Usually, transplant sites able to accommodate such large volumes of implants are confined to the peritoneal cavity (Sun et al., 1996; Elliott et al., 2005b; 2007; Cui et al., 2009), which is poorly vascularized and constitutes a preferential location for inflammatory and immunological reactions (Hsu et al., 1999; Omer 
et al., 2003a; de Groot et al., 2004). Thus, intraperitoneal injection seems less appropriate for promoting engraftment of encapsulated pig islets (Dufrane et al., 2006a).

To address these issues, investigators developed an approach to encapsulate islets by conformal coating, which may increase capsule stability, minimize capsule thickness and size and graft volume, and allow graft transplantation into the liver through the portal vein (Teramura and Iwata, 2008; 2009; Kizilel et al., 2010; Tomei et al., 2014). Conformal coating can be defined as the application of hydrogels to the surface of an islet cell by interfacial polymerization to form a cross-linked hydrogel and a thin $(<50 \mu \mathrm{m})$ coating (Cruise et al., 1998; Sefton et al., 2000; Wilson et al., 2008; Scharp and Marchetti, 2014). Islet surface modification with the biologically inert, amphiphilic polymer polyethylene glycol (PEG) has emerged as a promising and alternative approach. Not only did PEGylation have no adverse effects on islet morphology, viability, or functionality (Cruise et al., 1998; Contreras et al., 2004; Teramura and Iwata, 2009), but it was also found to prevent islet recognition by activated immune cells in vitro (Lee et al., 2004) and to reduce islet allograft damage or loss after intraportal transplantation (Teramura and Iwata, 2009). However, some in vivo studies suggested that islet surface modification alone, either with a PEG or heparin coating, was not a very stable immunoprotective method since combinatory treatments of lowdose immunosuppressants (e.g. cyclosporine or antico-stimulatory antibodies) had highly synergic effects on the maintenance of normoglycemia and inhibition of sensitized host immune responses (Lee et al., 2006a; 2006b; Jung et al., 2012; Jeong et al., 2013). It is unlikely that this technology will prove to be highly effective and applicable in pig islet xenotransplantation using present methods. Intraportal infusion of APIs (5000 IEQs/recipient) modified with PEG derivatives into non-obese diabetic/severe combined immune-deficient (NOD-SCID) mice gave better glucose control, but the euglycemia (non-fasting glucose $<200 \mathrm{mg} / \mathrm{dl}$ ) was very transient ( $<2$ weeks) (Contreras et al., 2004). In a study by Cabric et al. (2007), although transplantation of APIs (7500 IEQs/kg) coated with heparin into the livers of piglets resulted in lower insulin release (an indicator of cell damage), as well as decreased thrombin antithrombin (TAT) and $\mathrm{C} 3 \mathrm{a}$ generation, the observation period was too short $(<60 \mathrm{~min})$ and long-term graft viability/ functionality was not assessed.

Currently, by using the layer-by-layer (LbL) method, it is possible to fabricate complex coatings (e.g. PLL-g-PEG-biotin/streptavidin, chitosan/alginate, PEG$\mathrm{N}$-hydroxysuccinimide/alginate, or PEG-complement receptor 1 /heparin multilayer films) of nanometer thickness to significantly improve the stability of the layers, enhance nutrient diffusion, promote growth of new microvasculature, inhibit complement activation/blood-mediated inflammatory responses, and prolong islet graft survival (Cabric et al., 2007; Teramura and Iwata, 2008; Wilson et al., 2008; 2011; Zhi et al., 2012; Gattás-Asfura and Stabler, 2013; Luan and Iwata, 2013; Scharp and Marchetti, 2014). The main drawback of this innovative approach is the possible cytotoxicity of the compounds used. Thus, it is necessary to develop new methods to fabricate novel multifunctional coatings with excellent immunomodulatory capacities which can facilitate the reconstruction of the microenvironment (e.g. provide extracellular matrix support) and satisfy the physical demands of islet grafts. In this sense, the LbL strategy is still able to offer an opportunity to combine the inherent advantages of microencapsulation and conformal coating. In the future, the use of LbL with multifunctional materials derived from non-toxic biologically-active polymers or living regulatory cells will serve as a useful approach for sustained and favorable islet viability and functionality.

\section{Implantation sites}

The microenvironment of the implant site plays a major role in engraftment and survival of encapsulated pig islets xenografts. An optimal site should provide (1) a simple and safe implanting/removal operation, (2) immune protection, (3) a physiological route for insulin delivery, (4) a sufficient blood and oxygen supply, (5) enough space for a large volume of encapsulated islets, and (6) compatibility with immunoisolation systems (Zhu et al., 2014b).

Due to its lower restriction on the volume of grafts, intraperitoneal space has been used most often for the transplantation of encapsulated islets (Vériter et al., 2013), especially in xenotransplantations of 
encapsulated pig islets into experimental or preclinical models (Lanza et al., 1991; Sun et al., 1996; Kin et al., 2002; Omer et al., 2003a; Elliott et al., 2005a; 2005b; 2007; Vinerean et al., 2008; GrundfestBroniatowski et al., 2009). Although the procedure is easy and less invasive by laparoscopy, the peritoneal cavity may not be the ideal location for islet engraftment. Drawbacks include the lack of close contact with the bloodstream and the difficulty of retrieving the capsules if needed. Moreover, transplantation of encapsulated islets into the peritoneum may aggravate hypoxia and hamper the insulin secretory response since oxygen and insulin delivery through the peritoneal cavity is by passive transport only (de Groot et al., 2004). Studies in rodent models have demonstrated that macrophages and lymphocytes in the peritoneum are directly involved in the cellular infiltration, $\mathrm{PFO}$, and rapid degradation of the capsule (Omer et al., 2003a; Dufrane et al., 2006a; Vaithilingam et al., 2013). Despite the administration of immunosuppressive agents showing beneficial effects in improving the biocompatibility and prolonging the survival of encapsulated pig islets (either via macroencapsulation or microencapsulation) after intraperitoneal implantation (Omer et al., 2003a; Safley et al., 2005), the combination of immune suppression and encapsulation potentially reduces to nihil the interest in encapsulation (Orlando et al., 2014).

By contrast, subcapsular kidney (suitable only for microcapsules) and subcutaneous spaces (suitable for different encapsulation devices) showed weaker cellular reactions, better islet viability, and fewer broken capsules than the peritoneal cavity for transplantation of encapsulated pig islets (Dufrane et al., 2006a), rendering them interesting alternative sites for receiving encapsulated pig islets in preclinical studies (Elliott et al., 2005a; Dufrane et al., 2006b; 2010). Pre-vascularization of the implant site or co-encapsulation of pig islets and MSCs, especially for a subcutaneous space, is a very useful strategy to promote neovascularization around the implanted devices and to reduce hypoxic stress in the capsulated islets (Wang et al., 2002; 2003; Vériter et al., 2014). Nowadays, with the emergence of conformal coating and surface modification technology, the liver is again being investigated as a possible implant site for both allo- and xeno-islet grafts due to the resultant reduction in graft volume, prevention of instant blood-mediated inflammatory reaction (IBMIR) and intraportal thrombosis, and improvement in graft survival (Cabric et al., 2007; Teramura and Iwata, 2008; 2009; Luan and Iwata, 2013).

Other novel sites reported recently, such as striated muscle (Christoffersson et al., 2010; Espes et al., 2011) and bone marrow (Meier et al., 2014), represent feasible locations for the transplantation of microencapsulated or conformal coated islets with the advantages of good vascularization and relatively easy access. Nevertheless, further studies need to be performed in large animal models to evaluate the long-term graft survival and function in such sites.

\section{Conclusions}

Xenotransplantation of encapsulated pig islets may overcome the two major hurdles of conventional islet transplantation: limited human donor supply and the extensive use of immunesuppressants. Though studies conducted in both animal models and human recipients (early phase clinical trials) have demonstrated the feasibility of encapsulated pig islet xenontransplantation in the treatment of T1DM, clinical application is still a long way off. The successes are difficult to replicate (lab-to-lab variation) and there is a lack of a standard protocol for the preparation, engineering, and implantation of encapsulated islet xenografts. A consensus among specialists needs to be reached to further advance the current encapsulation technology. Among the encapsulation approaches, in our opinion, conformal coating of micron and submicron scale on individual islets or cell aggregates represents a promising direction. In the near future, with advances in genetically modified pig islets or stem-cell derived functional islets, improvements in encapsulation design and process, the emergence of novel biocompatible encapsulation materials and well bioengineered microenvironments for graft colonization, and greater preclinical and clinical experience with xenotransplantation will probably provide a clinically useful means of achieving $\beta$-cell replacement for IDDM.

\section{Acknowledgements}

The authors thank Dr. Ning JING (School of Medicine, Xi'an Jiaotong University, China) for help in editing this article. 


\section{Compliance with ethics guidelines}

Hai-tao ZHU, Lu LU, Xing-yu LIU, Liang YU, Yi LYU, and Bo WANG declare that they have no conflict of interest.

This article does not contain any studies with human or animal subjects performed by any of the authors.

\section{References}

Abalovich, A.G., Bacque, M.C., Grana, D., et al., 2009. Pig pancreatic islet transplantation into spontaneously diabetic dogs. Transplant. Proc., 41(1):328-330. [doi:10. 1016/j.transproceed.2008.08.159]

Bai, L., Tuch, B.E., Hering, B., et al., 2002. Fetal pig $\beta$ cells are resistant to the toxic effects of human cytokines. Transplantation, 73(5):714-722. [doi:10.1097/00007890200203150-00010]

Barkai, U., Weir, G.C., Colton, C.K., et al., 2013. Enhanced oxygen supply improves islet viability in a new bioartificial pancreas. Cell Transplant., 22(8):1463-1476. [doi:10. 3727/096368912X657341]

Beck, J., Angus, R., Madsen, B., et al., 2007. Islet encapsulation: strategies to enhance islet cell functions. Tissue Eng., 13(3):589-599. [doi:10.1089/ten.2006.0183]

Borg, D.J., Bonifacio, E., 2011. The use of biomaterials in islet transplantation. Curr. Diabetes Rep., 11(5):434-444. [doi:10.1007/s11892-011-0210-2]

Bottino, R., Balamurugan, A.N., Smetanka, C., et al., 2007. Isolation outcome and functional characteristics of young and adult pig pancreatic islets for transplantation studies. Xenotransplantation, 14(1):74-82. [doi:10.1111/j.13993089.2006.00374.x]

Bottino, R., Wijkstrom, M., van der Windt, D.J., et al., 2014. Pig-to-monkey islet xenotransplantation using multitransgenic pigs. Am. J. Transplant., 14(10):2275-2287. [doi:10.1111/ajt.12868]

Buder, B., Alexander, M., Krishnan, R., et al., 2013. Encapsulated islet transplantation: strategies and clinical trials. Immune Netw., 13(6):235-239. [doi:10.4110/in.2013.13. $6.235]$

Cabric, S., Sanchez, J., Lundgren, T., et al., 2007. Islet surface heparinization prevents the instant blood-mediated inflammatory reaction in islet transplantation. Diabetes, 56(8):2008-2015. [doi:10.2337/db07-0358]

Calafiore, R., Basta, G., 2014. Clinical application of microencapsulated islets: actual prospectives on progress and challenges. Adv. Drug Deliv. Rev., 67-68:84-92. [doi:10. 1016/j.addr.2013.09.020]

Casu, A., Bottino, R., Balamurugan, A.N., et al., 2008. Metabolic aspects of pig-to-monkey (Macaca fascicularis) islet transplantation: implications for translation into clinical practice. Diabetologia, 51(1):120-129. [doi:10. 1007/s00125-007-0844-4]

Christoffersson, G., Henriksnas, J., Johansson, L., et al., 2010. Clinical and experimental pancreatic islet transplantation to striated muscle: establishment of a vascular system similar to that in native islets. Diabetes, 59(10):25692578. [doi:10.2337/db10-0205]
Contreras, J.L., Xie, D., Mays, J., et al., 2004. A novel approach to xenotransplantation combining surface engineering and genetic modification of isolated adult porcine islets. Surgery, 136(3):537-547. [doi:10.1016/j.surg.2004. 05.031]

Cooper, D.K., Casu, A., 2009. The International Xenotransplantation Association consensus statement on conditions for undertaking clinical trials of porcine islet products in type 1 diabetes-Chapter 4: Pre-clinical efficacy and complication data required to justify a clinical trial. Xenotransplantation, 16(4):229-238. [doi:10.1111/j.13993089.2009.00543.x]

Cruise, G.M., Hegre, O.D., Scharp, D.S., et al., 1998. A sensitivity study of the key parameters in the interfacial photopolymerization of poly (ethylene glycol) diacrylate upon porcine islets. Biotechnol. Bioeng., 57(6):655-665. [doi:10.1002/(SICI)1097-0290(19980320)57:6<655::AI D-BIT3>3.0.CO;2-K]

Cui, H., Tucker-Burden, C., Cauffiel, S.M., et al., 2009. Long-term metabolic control of autoimmune diabetes in spontaneously diabetic nonobese diabetic mice by nonvascularized microencapsulated adult porcine islets. Transplantation, 88(2):160-169. [doi:10.1097/TP.0b013 e3181abbfc1]

de Groot, M., Schuurs, T.A., van Schilfgaarde, R., 2004. Causes of limited survival of microencapsulated pancreatic islet grafts. J. Surg. Res., 121(1):141-150. [doi:10. 1016/j.jss.2004.02.018]

de Vos, P., Spasojevic, M., Faas, M.M., 2010. Treatment of diabetes with encapsulated islets. Adv. Exp. Med. Biol., 670:38-53. [doi:10.1007/978-1-4419-5786-3_5]

de Vos, P., Lazarjani, H.A., Poncelet, D., et al., 2014. Polymers in cell encapsulation from an enveloped cell perspective. Adv. Drug Deliv. Rev., 67-68:15-34. [doi:10. 1016/j.addr.2013.11.005]

Dufrane, D., Gianello, P., 2012. Macro- or microencapsulation of pig islets to cure type 1 diabetes. World J. Gastroenterol., 18(47):6885-6893. [doi:10.3748/wjg.v18.i47.6885]

Dufrane, D., Goebbels, R.M., Fdilat, I., et al., 2005. Impact of porcine islet size on cellular structure and engraftment after transplantation: adult versus young pigs. Pancreas, 30(2):138-147. [doi:10.1097/01.mpa.0000147083.62501.4e]

Dufrane, D., Steenberghe, M., Goebbels, R.M., et al., 2006a. The influence of implantation site on the biocompatibility and survival of alginate encapsulated pig islets in rats. Biomaterials, 27(17):3201-3208. [doi:10.1016/j.biomater ials.2006.01.028]

Dufrane, D., Goebbels, R.M., Saliez, A., et al., 2006 b. Six-month survival of microencapsulated pig islets and alginate biocompatibility in primates: proof of concept. Transplantation, 81(9):1345-1353. [doi:10.1097/01.tp. 0000208610.75997.20]

Dufrane, D., Goebbels, R.M., Gianello, P., 2010. Alginate macroencapsulation of pig islets allows correction of streptozotocin-induced diabetes in primates up to 6 months without immunosuppression. Transplantation, 90(10):1054-1062. [doi:10.1097/TP.0b013e3181f6e267] 
Elliott, R.B., Escobar, L., Garkavenko, O., et al., 2000. No evidence of infection with porcine endogenous retrovirus in recipients of encapsulated porcine islet xenografts. Cell Transplant., 9(6):895-901.

Elliott, R.B., Escobar, L., Calafiore, R., et al., 2005a. Transplantation of micro- and macroencapsulated piglet islets into mice and monkeys. Transplant. Proc., 37(1):466-469. [doi:10.1016/j.transproceed.2004.12.198]

Elliott, R.B., Escobar, L., Tan, P.L., et al., 2005b. Intraperitoneal alginate-encapsulated neonatal porcine islets in a placebo-controlled study with 16 diabetic cynomolgus primates. Transplant. Proc., 37(8):3505-3508. [doi:10. 1016/j.transproceed.2005.09.038]

Elliott, R.B., Escobar, L., Tan, P.L., et al., 2007. Live encapsulated porcine islets from a type 1 diabetic patient $9.5 \mathrm{yr}$ after xenotransplantation. Xenotransplantation, 14(2): 157-161. [doi:10.1111/j.1399-3089.2007.00384.x]

Espes, D., Eriksson, O., Lau, J., et al., 2011. Striated muscle as implantation site for transplanted pancreatic islets. $J$. Transplant., 2011:352043. [doi:10.1155/2011/352043]

Foster, J.L., Williams, G., Williams, L.J., et al., 2007. Differentiation of transplanted microencapsulated fetal pancreatic cells. Transplantation, 83(11):1440-1448. [doi:10. 1097/01.tp.0000264555.46417.7d]

Gattás-Asfura, K.M., Stabler, C.L., 2013. Bioorthogonal layerby-layer encapsulation of pancreatic islets via hyperbranched polymers. ACS Appl. Mater. Interfaces, 5(20): 9964-9974. [doi:10.1021/am401981g]

Grundfest-Broniatowski, S.F., Tellioglu, G., Rosenthal, K.S., et al., 2009. A new bioartificial pancreas utilizing amphiphilic membranes for the immunoisolation of porcine islets: a pilot study in the canine. ASAIO J., 55(4):400-405. [doi:10.1097/MAT.0b013e3181a8deba]

Hatziavramidis, D.T., Karatzas, T.M., Chrousos, G.P., 2013. Pancreatic islet cell transplantation: an update. Ann. Biomed. Eng., 41(3):469-476. [doi:10.1007/s10439-0120676-3]

Hering, B.J., Wijkstrom, M., Graham, M.L., et al., 2006. Prolonged diabetes reversal after intraportal xenotransplantation of wild-type porcine islets in immunosuppressed nonhuman primates. Nat. Med., 12(3):301-303. [doi:10.1038/nm1369]

Hsu, B.R., Chang, F.H., Juang, J.H., et al., 1999. The rescue effect of 15-deoxyspergualin on intraperitoneal microencapsulated xenoislets. Cell Transplant., 8(3):307-315.

Jeong, J.H., Yook, S., Hwang, J.W., et al., 2013. Synergistic effect of surface modification with poly (ethylene glycol) and immunosuppressants on repetitive pancreatic islet transplantation into antecedently sensitized rat. Transplant. Proc., 45(2):585-590. [doi:10.1016/j.transproceed. 2012.02.028]

Jung, Y.S., Jeong, J.H., Yook, S., et al., 2012. Surface modification of pancreatic islets using heparin-DOPA conjugate and anti-CD154 mAb for the prolonged survival of intrahepatic transplanted islets in a xenograft model. Biomaterials, 33(1):295-303. [doi:10.1016/j.biomaterials.
2011.09.051]

Kim, H.I., Lee, S.Y., Jin, S.M., et al., 2009. Parameters for successful pig islet isolation as determined using 68 specific-pathogen-free miniature pigs. Xenotransplantation, 16(1):11-18. [doi:10.1111/j.1399-3089.2008.00504.x]

Kim, J.H., Kim, H.I., Lee, K.W., et al., 2007. Influence of strain and age differences on the yields of porcine islet isolation: extremely high islet yields from SPF CMS miniature pigs. Xenotransplantation, 14(1):60-66. [doi:10. 1111/j.1399-3089.2006.00364.x]

Kin, T., Nakajima, Y., Aomatsu, Y., et al., 2000. Humoral human xenoreactivity against isolated pig pancreatic islets. Surg. Today, 30(9):821-826. [doi:10.1007/s00595 0070065]

Kin, T., Iwata, H., Aomatsu, Y., et al., 2002. Xenotransplantation of pig islets in diabetic dogs with use of a microcapsule composed of agarose and polystyrene sulfonic acid mixed gel. Pancreas, 25(1):94-100. [doi:10.1097/ 00006676-200207000-00020]

Kirk, K., Hao, E., Lahmy, R., et al., 2014. Human embryonic stem cell derived islet progenitors mature inside an encapsulation device without evidence of increased biomass or cell escape. Stem Cell Res, 12(3):807-814. [doi:10. 1016/j.scr.2014.03.003]

Kizilel, S., Scavone, A., Liu, X., et al., 2010. Encapsulation of pancreatic islets within nano-thin functional polyethylene glycol coatings for enhanced insulin secretion. Tissue Eng. Part A, 16(7):2217-2228. [doi:10.1089/ten.tea.2009.0640]

Koulmanda, M., Qipo, A., Smith, R.N., et al., 2003. Pig islet xenografts are resistant to autoimmune destruction by non-obese diabetic recipients after anti-CD4 treatment. Xenotransplantation, 10(2):178-184. [doi:10.1034/j.13993089.2003.02040.x]

Kumagai-Braesch, M., Jacobson, S., Mori, H., et al., 2013. The theracyte device protects against islet allograft rejection in immunized hosts. Cell Transplant., 22(7):1137-1146. [doi:10.3727/096368912X657486]

Lanza, R.P., Butler, D.H., Borland, K.M., et al., 1991. Xenotransplantation of canine, bovine, and porcine islets in diabetic rats without immunosuppression. PNAS, 88(24): 11100-11104. [doi:10.1073/pnas.88.24.11100]

Lanza, R.P., Hayes, J.L., Chick, W.L., 1996. Encapsulated cell technology. Nat. Biotechnol., 14(9):1107-1111. [doi:10. 1038/nbt0996-1107]

Lanza, R.P., Jackson, R., Sullivan, A., et al., 1999. Xenotransplantation of cells using biodegradable microcapsules. Transplantation, 67(8):1105-1111. [doi:10.1097/ 00007890-199904270-00004]

Lee, D.Y., Nam, J.H., Byun, Y., 2004. Effect of polyethylene glycol grafted onto islet capsules on prevention of splenocyte and cytokine attacks. J. Biomater. Sci. Polym. Ed., 15(6):753-766. [doi:10.1163/156856204774196144]

Lee, D.Y., Park, S.J., Nam, J.H., et al., 2006a. A combination therapy of pegylation and immunosuppressive agent for successful islet transplantation. J. Control. Release, 110(2):290-295. [doi:10.1016/j.jconrel.2005.10.023] 
Lee, D.Y., Park, S.J., Nam, J.H., et al., 2006b. A new strategy toward improving immunoprotection in cell therapy for diabetes mellitus: long-functioning pegylated islets in vivo. Tissue Eng., 12(3):615-623. [doi:10.1089/ten.2006. 12.615]

Leitão, C.B., Cure, P., Tharavanij, T., et al., 2008. Current challenges in islet transplantation. Curr. Diabetes Rep., 8(4):324-331. [doi:10.1007/s11892-008-0057-3]

Luan, N.M., Iwata, H., 2013. Inhibition of instant bloodmediated inflammatory responses by co-immobilization of sCR1 and heparin on islets. Biomaterials, 34(21):50195024. [doi:10.1016/j.biomaterials.2013.03.041]

Ludwig, B., Zimerman, B., Steffen, A., et al., 2010. A novel device for islet transplantation providing immune protection and oxygen supply. Horm. Metab. Res., 42(13): 918-922. [doi:10.1055/s-0030-1267916]

Ludwig, B., Reichel, A., Steffen, A., et al., 2013. Transplantation of human islets without immunosuppression. PNAS 110(47):19054-19058. [doi:10.1073/pnas.1317561110]

Maki, T., Monaco, A.P., 1997. Porcine islet xenotransplantation utilizing a vascularized bioartificial pancreas. Ann. Transplant., 2(3):69-71.

Maki, T., Monaco, A.P., Mullon, C.J., et al., 1996. Early treatment of diabetes with porcine islets in a bioartificial pancreas. Tissue Eng., 2(4):299-306. [doi:10.1089/ten. 1996.2.299]

Malavasi, N.V., Rodrigues, D.B., Chammas, R., et al., 2010. Continuous and high-level in vivo delivery of endostatin from recombinant cells encapsulated in theracyte immunoisolation devices. Cell Transplant., 19(3):269-277. [doi:10.3727/096368909X480927]

Marigliano, M., Bertera, S., Grupillo, M., et al., 2011. Pig-to-nonhuman primates pancreatic islet xenotransplantation: an overview. Curr. Diabetes Rep., 11(5):402412. [doi:10.1007/s11892-011-0213-z]

Meier, R.P., Seebach, J.D., Morel, P., et al., 2014. Survival of free and encapsulated human and rat islet xenografts transplanted into the mouse bone marrow. PLOS ONE, 9(3):e91268. [doi:10.1371/journal.pone.0091268]

Nagaraju, S., Bottino, R., Wijkstrom, M., et al., 2015. Islet xenotransplantation: what is the optimal age of the isletsource pig? Xenotransplantation, 22(1):7-19. [doi:10.1111/ xen.12130]

Neufeld, T., Ludwig, B., Barkai, U., et al., 2013. The efficacy of an immunoisolating membrane system for islet xenotransplantation in minipigs. PLOS ONE, 8(8):e70150. [doi:10.1371/journal.pone.0070150]

O'Connell, P.J., Cowan, P.J., Hawthorne, W.J., et al., 2013. Transplantation of xenogeneic islets: are we there yet? Curr. Diabetes Rep., 13(5):687-694. [doi:10.1007/s11892013-0413-9]

O'Sullivan, E.S., Vegas, A., Anderson, D.G., et al., 2011. Islets transplanted in immunoisolation devices: a review of the progress and the challenges that remain. Endocr. Rev., 32(6):827-844. [doi:10.1210/er.2010-0026]

Omer, A., Keegan, M., Czismadia, E., et al., 2003a. Macro- phage depletion improves survival of porcine neonatal pancreatic cell clusters contained in alginate macrocapsules transplanted into rats. Xenotransplantation, 10(3): 240-251. [doi:10.1034/j.1399-3089.2003.01150.x]

Omer, A., Duvivier-Kali, V.F., Trivedi, N., et al., 2003b. Survival and maturation of microencapsulated porcine neonatal pancreatic cell clusters transplanted into immunocompetent diabetic mice. Diabetes, 52(1):69-75. [doi:10.2337/diabetes.52.1.69]

Orlando, G., Gianello, P., Salvatori, M., et al., 2014. Cell replacement strategies aimed at reconstitution of the $\beta$ cell compartment in type 1 diabetes. Diabetes, 63(5): 1433-1444. [doi:10.2337/db13-1742]

Petruzzo, P., Pibiri, L., de Giudici, M.A., et al., 1991. Xenotransplantation of microencapsulated pancreatic islets contained in a vascular prosthesis: preliminary results. Transpl. Int., 4(1):200-204. [doi:10.1111/j.1432-2277. 1991.tb01980.x]

Potter, K.J., Abedini, A., Marek, P., et al., 2010. Islet amyloid deposition limits the viability of human islet grafts but not porcine islet grafts. PNAS, 107(9):4305-4310. [doi:10. 1073/pnas.0909024107]

Prochorov, A.V., Tretjak, S.I., Goranov, V.A., et al., 2008. Treatment of insulin dependent diabetes mellitus with intravascular transplantation of pancreatic islet cells without immunosuppressive therapy. Adv. Med. Sci., 53(2):240-244. [doi:10.2478/v10039-008-0045-5]

Ramesh, A., Chhabra, P., Brayman, K.L., 2013. Pancreatic islet transplantation in type 1 diabetes mellitus: an update on recent developments. Curr. Diabetes Rev., 9(4):294311. [doi:10.2174/15733998113099990063]

Safley, S.A., Kapp, L.M., Tucker-Burden, C., et al., 2005. Inhibition of cellular immune responses to encapsulated porcine islet xenografts by simultaneous blockade of two different costimulatory pathways. Transplantation, 79(4): 409-418. [doi:10.1097/01.TP.0000150021.06027. DC]

Sakata, N., Sumi, S., Yoshimatsu, G., et al., 2012. Encapsulated islets transplantation: past, present and future. World J. Gastrointest. Pathophysiol., 3(1):19-26. [doi: 10.4291/wjgp.v3.i1.19]

Scalea, J., Hanecamp, I., Robson, S.C., et al., 2012. T-cellmediated immunological barriers to xenotransplantation. Xenotransplantation, 19(1):23-30. [doi:10.1111/j.13993089.2011.00687.x]

Scharp, D.W., Marchetti, P., 2014. Encapsulated islets for diabetes therapy: history, current progress, and critical issues requiring solution. Adv. Drug Deliv. Rev., 67-68: 35-73. [doi:10.1016/j.addr.2013.07.018]

Sefton, M.V., May, M.H., Lahooti, S., et al., 2000. Making microencapsulation work: conformal coating, immobilization gels and in vivo performance. J. Control. Release, 65(1-2):173-186. [doi:10.1016/S0168-3659(99)00234-5]

Semaan, M., Kaulitz, D., Petersen, B., et al., 2012. Long-term effects of PERV-specific RNA interference in transgenic pigs. Xenotransplantation, 19(2):112-121. [doi:10.1111/j. 1399-3089.2012.00683.x] 
Shapiro, A.M., Lakey, J.R., Ryan, E.A., et al., 2000. Islet transplantation in seven patients with type 1 diabetes mellitus using a glucocorticoid-free immunosuppressive regimen. N. Engl. J. Med., 343(4):230-238. [doi:10.1056/ NEJM200007273430401]

Shin, J.S., Kim, J.S., Kim, J.M., et al., 2014. Minimizing immunosuppression in islet xenotransplantation. Immunotherapy, 6(4):419-430. [doi:10.2217/imt.14.14]

Shin, S., Shin, J.E., Yoo, Y.J., 2013. Attachment of alginate microcapsules onto plasma-treated PDMS sheet for retrieval after transplantation. Biotechnol. Appl. Biochem., 60(6):617-622. [doi:10.1002/bab.1124]

Sörenby, A.K., Kumagai-Braesch, M., Sharma, A., et al., 2008. Preimplantation of an immunoprotective device can lower the curative dose of islets to that of free islet transplantation: studies in a rodent model. Transplantation, 86(2):364-366. [doi:10.1097/TP.0b013e31817efc78]

Sun, Y., Ma, X., Zhou, D., et al., 1996. Normalization of diabetes in spontaneously diabetic cynomologus monkeys by xenografts of microencapsulated porcine islets without immunosuppression. J. Clin. Invest., 98(6):1417-1422. [doi:10.1172/JCI118929]

Tan, P.L., 2010. Company profile: tissue regeneration for diabetes and neurological diseases at living cell technologies. Regen. Med., 5(2):181-187. [doi:10.2217/rme.10.4]

Teramura, Y., Iwata, H., 2008. Islets surface modification prevents blood-mediated inflammatory responses. Bioconjug. Chem., 19(7):1389-1395. [doi:10.1021/bc800064t]

Teramura, Y., Iwata, H., 2009. Surface modification of islets with peg-lipid for improvement of graft survival in intraportal transplantation. Transplantation, 88(5):624-630. [doi:10.1097/TP.0b013e3181b230ac]

Thompson, P., Cardona, K., Russell, M., et al., $2011 \mathrm{a}$. CD40-specific costimulation blockade enhances neonatal porcine islet survival in nonhuman primates. Am. J. Transplant., 11(5):947-957. [doi:10.1111/j.1600-6143. 2011.03509.x]

Thompson, P., Badell, I.R., Lowe, M., et al., 2011b. Islet xenotransplantation using gal-deficient neonatal donors improves engraftment and function. Am. J. Transplant., 11(12):2593-2602. [doi:10.1111/j.1600-6143.2011.03720.x]

Thompson, P., Badell, I.R., Lowe, M., et al., 2012. Alternative immunomodulatory strategies for xenotransplantation: CD40/154 pathway-sparing regimens promote xenograft survival. Am. J. Transplant., 12(7):1765-1775. [doi:10. 1111/j.1600-6143.2012.04031.x]

Tomei, A.A., Manzoli, V., Fraker, C.A., et al., 2014. Device design and materials optimization of conformal coating for islets of langerhans. PNAS, 111(29):10514-10519. [doi:10.1073/pnas.1402216111]

Vaithilingam, V., Fung, C., Ratnapala, S., et al., 2013. Characterisation of the xenogeneic immune response to microencapsulated fetal pig islet-like cell clusters transplanted into immunocompetent C57BL/6 mice. PLoS ONE, 8(3):e59120. [doi:10.1371/journal.pone.0059120]

Valdés-González, R.A., Dorantes, L.M., Garibay, G.N., et al.,
2005. Xenotransplantation of porcine neonatal islets of Langerhans and Sertoli cells: a 4-year study. Eur. J. Endocrinol., 153(3):419-427. [doi:10.1530/eje.1.01982]

Valdés-González, R.A., White, D.J., Dorantes, L.M., et al., 2007. Three-yr follow-up of a type 1 diabetes mellitus patient with an islet xenotransplant. Clin. Transplant., 21(3):352-357. [doi:10.1111/j.1399-0012.2007.00648.x]

Valdes-Gonzalez, R., Rodriguez-Ventura, A.L., White, D.J., et al., 2010. Long-term follow-up of patients with type 1 diabetes transplanted with neonatal pig islets. Clin. Exp. Immunol., 162(3):537-542. [doi:10.1111/j.1365-2249.2010. 04273.x]

van der Windt, D.J., Bottino, R., Casu, A., et al., 2009. Long-term controlled normoglycemia in diabetic nonhuman primates after transplantation with hCD46 transgenic porcine islets. Am. J. Transplant., 9(12):2716-2726. [doi:10.1111/j.1600-6143.2009.02850.x]

van Schilfgaarde, R., de Vos, P., 1999. Factors influencing the properties and performance of microcapsules for immunoprotection of pancreatic islets. J. Mol. Med. (Berl.), 77(1):199-205. [doi:10.1007/s001090050336]

Vériter, S., Gianello, P., Dufrane, D., 2013. Bioengineered sites for islet cell transplantation. Curr. Diabetes Rep., 13(5):745-755. [doi:10.1007/s11892-013-0412-x]

Vériter, S., Gianello, P., Igarashi, Y., et al., 2014. Improvement of subcutaneous bioartificial pancreas vascularization and function by coencapsulation of pig islets and mesenchymal stem cells in primates. Cell Transplant., 23(11):1349-1364. [doi:10.3727/096368913X663550]

Vinerean, H.V., Gazda, L.S., Hall, R.D., et al., 2008. Improved glucose regulation on a low carbohydrate diet in diabetic rats transplanted with macroencapsulated porcine islets. Cell Transplant., 17(5):567-575. [doi:10.3727/09636890 8785095962]

Wang, W., Gu, Y., Tabata, Y., et al., 2002. Reversal of diabetes in mice by xenotransplantation of a bioartificial pancreas in a prevascularized subcutaneous site. Transplantation, 73(1):122-129. [doi:10.1097/00007890-2002 01150-00023]

Wang, W., Gu, Y., Hori, H., et al., 2003. Subcutaneous transplantation of macroencapsulated porcine pancreatic endocrine cells normalizes hyperglycemia in diabetic mice. Transplantation, 76(2):290-296. [doi:10.1097/01.TP.000 $0073613.25658 .4 \mathrm{D}]$

Weir, G.C., 2013. Islet encapsulation: advances and obstacles. Diabetologia, 56(7):1458-1461. [doi:10.1007/s00125-0132921-1]

Wilson, J.T., Cui, W., Chaikof, E.L., 2008. Layer-by-layer assembly of a conformal nanothin PEG coating for intraportal islet transplantation. Nano Lett., 8(7):1940-1948. [doi:10.1021/n1080694q]

Wilson, J.T., Cui, W., Kozlovskaya, V., et al., 2011. Cell surface engineering with polyelectrolyte multilayer thin films. J. Am. Chem. Soc., 133(18):7054-7064. [doi:10. 1021/ja110926s]

Wynyard, S., Nathu, D., Garkavenko, O., et al., 2014. 
Microbiological safety of the first clinical pig islet xenotransplantation trial in New Zealand. Xenotransplantation, 21(4):309-323. [doi:10.1111/xen.12102]

Yonekawa, Y., Matsumoto, S., Okitsu, T., et al., 2005. Effective islet isolation method with extremely high islet yields from adult pigs. Cell Transplant., 14(10):757-762. [doi:10.3727/000000005783982512]

Zhi, Z.L., Kerby, A., King, A.J., et al., 2012. Nano-scale encapsulation enhances allograft survival and function of islets transplanted in a mouse model of diabetes. Diabetologia, 55(4):1081-1090. [doi:10.1007/s00125-0112431-y]

Zhu, H.T., Yu, L., Lyu, Y., et al., 2014a. Optimal pig donor selection in islet xenotransplantation: current status and future perspectives. J. Zhejiang Univ.-Sci. B (Biomed. \& Biotechnol.), 15(8):681-691. [doi:10.1631/jzus.B1400120]

Zhu, H.T., Wang, W.L., Yu, L., et al., 2014b. Pig-islets xenotransplantation: recent progress and current perspectives. Front. Surg., 1:7. [doi:10.3389/fsurg.2014.00007]

Zimmermann, H., Shirley, S.G., Zimmermann, U., 2007.
Alginate-based encapsulation of cells: past, present, and future. Curr. Diabetes Rep., 7(4):314-320. [doi:10.1007/ s11892-007-0051-1]

\section{中文概要}

题 目: 胶囊猪胰岛移植治疗糖尿病: 现况与进展

概 要: 本文对胶囊猪胰岛异种移植治疗糖尿病的相关研 究进行综述, 展示目前的研究现状, 指出目前存 在的问题, 并提出未来的研究方向。基于包被胰 岛的体积、活性、功能及生物安全性等重要决定 因素, 对猪胰岛来源、胶囊包被技术/方法及植入 部位的选择进行了对比性、系统性地阐述, 具有 较高的参考价值。胶囊猪胰岛异种移植治疗糖尿 病具有良好的应用前景, 小胶囊或保形包被技术 或是今后的发展方向。

关键词: 胶囊包被; 猪; 胰岛; 异种移植; 糖尿病 